\title{
Effect of phenolic compounds on nematodes- A review
}

\author{
Puja Ohri* and Satinder K aur Pannu \\ Department of Zoology, Guru Nanak Dev University, Amritsar-143005, INDIA \\ *Corresponding author. E-mail: ohri_puja_11@ rediffmail.com
}

\begin{abstract}
The term, phenolics has been used to describe a group of structurally diverse plant secondary metabolites. This group includes metabolites derived from the condensation of acetate units (terpenoids), those produced by the modification of aromatic amino acids (phenylpropanoids, cinnamic acid, lignin precursor, catechols and coumarins), flavonoids, isoflavonoids, and tannins. The occurrence and metabolism of phenolic substances in plants, in response to injury or invasion by pathogens, such as fungi, bacteria and viruses have already been studied. Oxidised compounds produced in plants after invasion by pathogens often show considerable biological activity and are a common mechanism of resistance to plant pathogens. The present review gives information regarding the effects of different phenolic compounds on nematode system. It is found that these compounds are involved in plant defense and hence provide resistance against nematode attack.
\end{abstract}

Keywords: Flavonoids, Phenols, Phenylpropanoids, Salicylic acid, Tannins, Nematodes

\section{INTRODUCTION}

Plants produce a wide range of biologically active chemicals, secondary metabolites, which are involved in plant defense against pests and diseases. The major classes of secondary metabolites include Alkaloids, Terpenoids and Phenolic compounds. Among the three groups, Phenolic compounds have been used to describe a group of structurally diverse plant secondary metabolites. Phenolic compounds possess in common an aromatic ring bearing one or more hydroxyl substituents. Most of the phenolic compounds are polyphenols and they are categorized into 5 groups:Simple phenols and phenolic acids, phenylpropanoids, flavonoids, tannins and quinone. The occurrence and metabolism of phenolic substances in plants, in response to injury or invasion by pathogens, such as fungi, bacteria and viruses, have been studied extensively (Farkas and Kiraly, 1962; Patil et al., 1964). In nematodes too, a large number of phenolic compounds have been found to have strong nematicidal activity. The present review is an effort to consolidate the literature available regarding the effect of these compounds on nematodes.

\section{PHENOLS}

A number of phenolic compounds including monohydroxy, dihydroxy and trihydroxy compounds, quinones and aromatic acids such as transcinnamic acid have been studied for their nematicidal activity and their effect on egg hatch of M eloidogyne incognita (Mahajan et al., 1985). Transcinnamic acid, pyrogallol, 2-OH napthoic acid and ethyl gallate were found to be highly toxic with mortality greater than $95 \%$. Whereas 2,6 dibromoquinone chloroimide, catechin hydrate, $\beta$ resorcylic acid, m-hydroxy benzoic acid and 3,4dihydroxy benzoic acid exhibited moderate activity with mortality ranging from $52-66 \%$. In the same study, ten phenolic compounds were oxidized and assayed for nematicidal activity out of which oxidized $\alpha$-resorcylic acid, ferulic acid, 3,4-dihydroxybenzoic acid and caffeic acid showed high mortality effect. Lower activity was indicated by chlorogenic acid and catechin hydrate, while vanillic acid, phloroglucinol and p-hydroxy cinnamic acid induced negligible nematode mortality. Naringenin was found to be the most effective, with total suppression of hatching. 2-OH napthoic acid and transcinnamic acid were also highly effective in suppressing the egg hatch. Again in year 1992, Mahajan et al. tested a wide range of phenolic compounds for their nematicidal activity against $M$. incognita. Out of the 55 phenolic compounds tested, coumestrol, juglone, dihydroxy caffeic acid quinone, 2,6dihydroxy benzoic acid, gentisic acid, p-methoxycinnamic acid, 3-phenylphenol, 7-OH-coumarin, vanillic, syringic and protocatechuic acid showed high nematicidal activity.

Host-parasite relationships of $M$. incognita acrita and Pratylenchus penetrans have been compared on three closely related cultivars of tomato: 'Nemared', resistant to root knot nematodes, 'Hawaii 7153', moderately resistant and 'B-5', susceptible (Hung and Rohde, 1973). It was reported that the large number of larvae of $M$. incognita and P. penetrans never penetrated the resistant variety of tomato due to some sort of inhibition and this 
inhibition was provided by phenolic compounds. Chlorogenic acid was identified as the major phenolic compound in the roots after or before infection. The nematicidal activity of various substituted phenols, phenoxyacetic acid esters and hydrazides was also determined against second stage juveniles of seed-gall nematode (Anguina tritici), root knot nematode ( $M$. javanica) and pigeon pea cyst nematode ( $\mathrm{H}$. cajani) to establish the relationship of activity with structural variations (Malik et al., 1989). They reported that phenols with electron donating substituents, particularly the chloro-substituted phenols are more active than those with electron withdrawing substituents.

The effects of naturally occurring napthoquinonesplumbagin, juglone and lawsone extracted from Plumbago zeylanica, J uglans regia and Lawsonia alba respectively has been studied in vitro on root-knot nematode, $M$. javanica (Dama, 2002). Nematodes were exposed at concentration $200 \mathrm{mg} / 10 \mathrm{ml}$ water/flask for $6 \mathrm{~h}, 12 \mathrm{~h}$ and $24 \mathrm{~h}$. It was found that percent mortality was $100 \%$ in plumbagin, $97.9 \%$ in juglone and $58.3 \%$ in lawsone. The structure-activity relationship observed for the action of plumbagin on $M$. incognita demonstrated quite a rigid structural requirement for attainment of maximal activity. Thus, juglone and lawsone, differ from plumbagin only in absence of a methyl group, were considerably less effective against M . javanica. Similar earlier studies also showed that plumbagin had toxic effect on development of parasitic nematode, $\mathrm{H}$ aemonchus contortus (Fetterer and Fleming, 1991) and juglone has been implicated as an antiparasitic agent (Dama and Jadhav, 1997).

\section{SALICYLIC ACID}

Salicylic acid (SA) is a natural phenolic compound present in many plants and is involved in induction of resistance in various plants. This has been reported by Ganguly et al. (1999) in which the foliar application of salicylic acid on tomato cv. Pusa Ruby @ 25 and $50 \mu \mathrm{g} / \mathrm{ml}$ affected the development of M. incognita. The development of juveniles into adult females was considerably delayed and population of males increased indicating sex reversal, on application of $50 \mu \mathrm{g} / \mathrm{ml}$ of SA. Reduced root galling (50\% or lower) was observed in treated tomato plants over control.

The effect of exogenous salicylic acid on the M . incognita infesting tomato Pusa Ruby has been worked out by Nandi et al. (2000a). The result showed that SA increased plant growth in terms of shoot length, shoot weight and root length as compared to the inoculated untreated plants. Root galls, nematode population in root as well as in soil and root protein content were significantly reduced in SA treated plants as compared to the untreated ones. Pretreatment with SA showed better plant growth and lesser intensity of root-knot disease as compared to the post-inoculation treatment. Similar results were obtained when the effect of SA was explored on M. incognita infesting cowpea and okra (Nandi et al., 2000b, 2002,2003).

Pankaj and Sharma (2003) demonstrated relative sensitivity of $M$. incognita and Rotylenchus reniformis to salicylic acid on okra. Salicylic acid when sprayed promoted the plant growth significantly over control. The number of galls and egg masses produced by $M$. incognita was significantly low. Thus SA not only interfered in gall formation but also impaired the production of normal egg masses and subsequently hatching.

Sirohi and Pankaj (2005) has conducted a study on bare root dip application of systemic acquired resistance (SAR) inducing chemicals in tomato against $M$. incognita. In the study, three week old tomato seedlings (cv. Pusa Ruby) were treated with salicylic acid or 2hydroxybenzoic acid, rose bengal and gibberellic acid @ 25,50 and $100 \mu \mathrm{g} / \mathrm{ml}$ as foliar spray, root dip for $10 \mathrm{~min}$ and soil drench with simultaneous or delayed nematode inoculation. The tomato seedlings were harvested after 60 days from date of sowing. The results showed that all three chemicals had varied effect as resistance inducers and of all three chemicals SA was the most effective molecule for inducing resistance while other two chemicals also showed same effect as inducers for resistance but the effect was not significant. In another study by Pankaj et al. (2005) on the estimation of salicylic acid and its role in resistance mechanism in chickpea against M . incognita showed that SA has a positive role in resistant mechanism in chickpea. Similarly, a study on the evaluation of SA as SAR inducer against $M$. incognita on tomato cv. $\mathrm{Co} 3$ has been conducted by Jayakumar et al. (2006). It was demonstrated that among the various dosage levels and methods of application of SA i.e. root dip and foliar application of SA on tomato cv. Co3@ 50,100 and 200 ppm affected the development of $M$. incognita and significantly increased plant height, root length, shoot weight and root weight at 90 days after transplanting. Hence, it was concluded that such a plant growth promoting effect might have induced by exogenous application of SA and reduction in root-knot nematode population is mainly due to resistance induced by it.

The effect of salicylic acid on the M . incognita has been demonstrated by Naik and Sharma (2007) in relation to eight pesticides (Metayalaxyl + Mancozeb, Thiophanate methyl, Mancozeb, Bitertanol, Phenomiphos, Phorate, Thionazin and Carbofuran) and a growth hormone Indole3 -acetic acid. Effect on egg hatching and larval mortality was observed. Two concentrations viz., $0.1 \%$ and $0.2 \%$ were used where it was observed that salicylic acid at a concentration of $0.2 \%$ was more effective than $0.1 \%$ Recently, Wubben et al. (2008) utilized A. thaliana mutants in SA biosynthesis and signal transduction to investigate the role of SA in inhibiting parasitism by the 
beet cyst nematode $\mathrm{H}$. schachtii. It was determined that SA-deficient mutants (sid2-1, pad 4-1 and NahG) exhibited increased susceptibility to $H$. schachtii, whereas the npr1 suppressor mutation snil showed decreased susceptibility. Also, constitutive pathogenesis-related (PR) gene expressing mutants (cpr1 and cpr6) did not show altered susceptibility to $H$. schachtii; however, constitutive PR gene expression was restricted to cpr1 shoots with wild-type levels of PR-1 transcript present in cpr1 roots. Furthermore, it was determined that $\mathrm{H}$. schachtii infection elicits SA-independent PR-2 and PR5 induction in wild-type roots, while PR-1 transcript and total SA levels remained unaltered. Thus, it was concluded that SA acts via NPR1 to inhibit nematode parasitism which, in turn, was negatively regulated by SNI1. The results also showed an inverse correlation between root basal PR-1 expression and plant susceptibility to $H$. schachtii and suggested that successful cyst nematode parasitism might involve a local suppression of SA signaling in roots.

\section{PHENYLPROPANOID}

The phenylpropanoids serve as low molecular weight flower pigments, antibiotics, UV protectants, insectrepellants and signal molecules in plant-microbe interaction. Phenylpropanoids are reported to take part in the wound and defense responses of plants. The biosynthetic pathway of the phenylpropanoids is well characterized and constitutes a potential target for the improvement of resistance against nematodes. Enzymes directly or indirectly functional in the phenylpropanoid pathway are induced in plants in response to wounding and infection by pathogens, including sedentary endoparasitic nematodes.

The activities of phenylalanine ammonia lyase (PAL), peroxidase (PO), polyphenoloxidase (PPO) have been analyzed in banana roots before and 1, 3 and 7 days after inoculation with the burrowing nematode Radopholus similis and in comparison with mechanically wounded roots (Wuyts et al., 2006a). Constitutive activities of PAL, $\mathrm{PO}$ and PPO were lower in the resistant cv. Yangambi $\mathrm{km5}$ (M usa acuminata AAA) than in the susceptible cv. Grande Naine (M . acuminata AAA). PAL was induced in R. similis- inoculated roots of the resistant cultivar only and this response was different from wound induction. For PO and PPO, levels in the resistant cultivar increased to those of the susceptible cultivar as a response to general stress in the plants during the experiment. Nevertheless, final levels of PO and PPO activity in R. similis-inoculated roots of the resistant cultivar were significantly higher than constitutive ones. Also, a broad-spectrum evaluation has been made on effects of phenylpropanoids (simple phenolics and flavonoids) and selected monoterpenoids and alkaloids on the behaviour of the migratory endoparasites
Radopholus similis and P. penetrans and the sedentary endoparasite M. incognita (Wuyts et al., 2006b). In vitro bioassays assessed effects on chemotaxis, motility, viability and hatch. Compared with the other two nematode species, P. penetrans was remarkably insensitive to the test compounds. Only phloretin was hatch inhibitive. This property was shared by other chalcone-related compounds for R. similis. Repellents and motility inhibitors for R. similis and M. incognita were found among the simple phenolic compounds. Flavonols stood out as repellent compounds for both these nematode species, while they were, in their degraded form, also motility inhibitors for M. incognita. Dopamine was an attractant for R. similis, while ferulic acid was strongly motility inhibitive and toxic $\left(\mathrm{LC}_{50}\right.$ of $120 \mathrm{mg} / \mathrm{ml}$ ) for this nematode species. Salicylic acid was a strong attractant for $M$. incognita. The compound was also nematicidal $\left(\mathrm{LC}_{50}\right.$ of $\left.46 \mathrm{mg} / \mathrm{ml}\right)$ and an irreversible inhibitor of hatch. Further, in another study by Wuyts et al. (2007), healthy and infected roots of two susceptible (Grande naine and Obino l'ewai) and three resistant cultivars (Yangambi km5, Pisang jari buaya and Calcutta 4) were extracted and chemically analyzed for their lignin content and phenylpropanoid profile. Compared to the susceptible cultivars, the resistant cultivars constituted significantly higher levels of lignin in the vascular bundle and cell wall bound ferulic acid esters in the cortex. Infection-induced lignification was observed in the vascular bundles of all cultivars. The catecholamine dopamine was identified as a major metabolite in banana roots.

Several other studies have been conducted where the nematode induced synthesis of PAL, PPO and total phenols were observed. Three pigeonpea varieties, with variable reactions to the reniform nematode, viz; 556-1 (resistant), Mukta (moderately susceptible) and 3430-1 (susceptible) were selected for the estimation of total phenols (Thakar and Yadav, 1986). The results indicated the presence of high total phenol content in the resistant variety $(0.86 \mathrm{mg} / \mathrm{g}$ fresh roots $)$ as compared to $(0.27 \mathrm{mg} /$ $\mathrm{g}$ fresh roots) susceptible variety 3430-1. It was suggested that increased phenolic level in 556-1 might be the reason for its resistance to the reniform nematode. Few years back, Begum and Sivakumar (2004) carried out biochemical analysis for the estimation of total phenols and peroxidase activity in which two biocontrol agents vitz., P seudomonas fluorescens, Trichoderma viride were used both as seed treatment (ST)@ 10g/kg seeds and 40/ $\mathrm{kg}$ seeds respectively and soil application (SA) @ 2.5kg/ ha. The results showed that phenol content and peroxidase activity were significantly higher in the combination treatment of $P$. fluorescens (ST) and Neem cake (SA) followed by combination treatment of $\mathrm{T}$. vi ride (ST) and Neem cake (SA).

One of the studies conducted by Sirohi and Dasgupta 
(1993) provide evidence for early induction of PAL and its relationship with resistance expression in Cowpea cv. C-152 inoculated with root knot nematode $M$. incognita race 1 . The rate of increase of PAL and it's activity between 24-72 hours was extensively higher in inoculated resistant cowpea cv. C-152 in contrast to it's control as well as to inoculated and uninoculated susceptible cowpea cv. Pusa do Fasli (PDF), connoting that the nematode-mediated biomolecular defense mechanism was activated as early as 24 hours after inoculation in resistant cowpea cultivar. It was inveterately demonstrated that Chlorogenic acid (CGA) turnover values were higher in between 24-72 hours in inoculated resistant cultivar C152 compared to inoculated susceptible cultivar PDF implying that the onset of faster rate of PAL in inoculated resistant $\mathrm{C}-152$ preceded highest concentration of CGA which was not the case in PDF, indicating an early, faster onset or completion of lignification in C-152 as compared to PDF. Along with the PAL, 4-hydroxycinnamic acid: CoA ligase forms the critical enzyme required for the synthesis and subsequent distribution of phenylpropanoid phenols. The specific activity of 4hydroxycinnamic acid: CoA ligase was assayed by Pankaj et al. (2001) using p-coumaric acid as a substrate at an early stage of infection with $M$. incognita in both susceptible (Pusa Ruby) and resistant (Nemamukt and Hisar lalit) cultivars of tomato. The result indicated that the activity was higher in resistant cultivars at different time intervals compared to susceptible one thus inferring an important role of CoA ligase in resistant reaction in tomato against $M$. incognita.

Race specific biochemical responses to the host differentials were also used for identification of races of M. incognita by Swain et al. (2004). Investigations on sequential development of PAL, PPO, phenol and lignin like polymers were undertaken in differential host plants (cotton cv. Deltapine-16 and tobacco cv. NC-95) along with susceptible host (cotton cv. H-777 and tobacco cv. FCV-special) after inoculation with different host races of $M$. incognita. All the races induced a faster and early accumulation of these defense parameters upon inoculation to host differentials than their healthy controls, whereas race-inoculated susceptible tobacco cv. FCV-special and cotton cv. H-777 showed a gradual and delayed accumulation in their defense reactions, but the percent increase over their uninoculated controls, was of less magnitude than that observed in host differentials at different time intervals.

\section{TANNINS}

Tannins are a group of water-soluble polyphenols that are able to precipitate proteins, such as gelatin, from solution. They are found in a large variety of higher plants of both herbaceous and woody type. Substantial accumulations of vegetable tannins may be found in almost any part of a plant. Tannins protect some plants against herbivores (Feeny, 1976), and they are toxic to a wide variety of fungi, bacteria and yeasts (Scalbert, 1991). Studies on the effects of tannins on plant parasitic nematodes are few since it causes severe phytotoxicity to the host plant. The effectiveness of four common organic amendments with high phenolic and tannin content has been investigated for the control of $M$. arenaria (Neal) Chitwood (Mian and Rodriguez-Kabana, 1982). The number of galls/gm of fresh root were reduced by applications of tannic acid of $0.4 \%$ or higher and a similar response was observed for gall index values. All applications of tannic acid at rates of $0.2 \%$ or higher resulted in shorter shoots, lower shoot and root weights, and lower values for the root condition index than the values corresponding to plants from untreated soils. Tannic acid had no effect on the number of seedlings that emerged. In another study, Chen et al. (1997) had evaluated the effects of tannic acid on hatching of the soybean cyst nematode, Heterodera glycines and to determine the possibility of using the compound to induce nematodes to hatch. It was found that low concentrations of tannic acid increased nematode hatching. Percent hatch increased with an increase of tannic acid concentration up to 35 to $40 \mathrm{mg} / \mathrm{liter}$ and then declined with increasing concentration i.e. high concentration of tannic acid inhibit hatching. Similarly the efficacy of tannic acid has been studied against $M$. arenaria on tomato along with its effects on the behavior of $M$. arenaria, $M$. incognita, $H$. glycines, and R. similis (Hewlett et al., 1997). Tannic acid was found to reduce galling as compared to the untreated control, regardless of methods of application. In behavior studies on water agar, M eloidogyne $\mathrm{J}_{2} \mathrm{~s}$ were attracted to areas with an increasing tannic acid gradient. R. similis was repelled from the tannic acid gradient in one of two experiments but there was no effect on $\mathrm{H}$. glycines. The behavioral response of Meloidogyne $\mathrm{J}_{2} \mathrm{~s}$ to tannic acid indicated that tannins and other polyphenolic compounds may be chemical cues that Meloidogyne spp. use to navigate toward roots, recognize plant hosts or locate areas for root penetration.

\section{FLAVONOIDS}

Flavonoids are low molecular weight secondary metabolites that have many diverse functions including defense, UV protection, auxin transport inhibition, allelopathy and flower coloring.

It is evident that nematode resistant plants have higher constitutive levels of transcripts for key enzymes involved in biosynthesis of isoflavonoid phytoalexins, which are known to play a role in fungal resistance and are implicated in resistance to both sedentary and migratory nematodes. In a study by Baldridge et al. (1998), preliminary evidence was obtained for the involvement 
of several known plant defense response genes in alfalfa resistance to $P$. penetrans. The validity of phenypropanoid pathway enzyme transcript suppression in $P$. penetrans-infected resistant alfalfa plants was supported by the simultaneous induction of b-1,3glucanase transcripts in a resistant versus susceptible host pattern typical of many other plant/pathogen systems. Earlier also, it has been reported that the roots of resistant soybeans inoculated with $M$. incognita exhibit a hypersensitive response and accumulate the phytoalexin glyceollin, a product of the isoflavonoid branch of the phenylpropanoid pathway. Also, Glyceollin inhibit oxidation, respiration and motility of $M$. incognita in vitro and accumulate immediately adjacent to the head region of soybean cyst nematode ( $H$. glycines) in resistant but not susceptible soybean root tissue (Kaplan et al., 1980). In 1998, Gonzalez and Estevez-Braun demonstrated the nematicidal and hatching inhibition activities of (E)-chalcone (trans-1,3-diphenylpropenone), for potato-cyst nematodes Globodera pallida and G. rostochiensis. The nematicidal activity of (E)-chalcone was very high $\left(\mathrm{LC}_{50}=33 \mathrm{mM}, \mathrm{LC}_{100}<50 \mathrm{mM}\right)$ and $(\mathrm{E})$ chalcone was able to inhibit the hatching of second-stage juveniles from nematode cysts. Earlier to these studies, high activity against the zooparasitic nematodes Syphacia obvelata and Nematospiroides dubius had also been found for (E)-chalcone and other related compounds (Laliberte et al., 1967). Several other examples of nematotoxic isoflavonoids include- the widely distributed flavonoid quercetin, which inhibited reproduction of $M$. javanica as a soil drench at $400 \mathrm{mg} / \mathrm{ml}$ (Osman and Viglierchio, 1988); the flavone glycosides linaroside and lantanoside from L. camara, which were lethal to $M$. incognita juveniles at 1.0\% (Begum et al., 2000) and ratenone, which displayed nematicidal activity towards C. elegans (Stadler et al., 1994).

Even though it is known that nematode infection adversely affects nodulation, it was not known if this interaction also affects isoflavonoid production. So the effect of $\mathrm{H}$. glycines infection on isoflavonoid production was studied by Kennedy et al. in 1999 . The effects of infection on nodulation were examined in soybean cultivars, susceptible and resistant to $\mathrm{H}$. glycines. Different races of $H$. glycines had different effects on soybean nodulation. Race 1 reduced nodulation and nitrogen fixing capacity and caused severe chlorosis on soybean. In contrast, race 2 and 4 did not affect the growth of soybean severely. Soriano et al. (2004) examined the inducibility of possible defense compounds in oats in response to invasion by parasitic nematodes with different feeding behavior. They reported that oats (Avena sativa) are highly resistant to pest and diseases. Oats possesses secondary metabolites that impart resistance to nematodes. Three flavone-C-glycoside, O-
methyl-apigenin-C-hexoside-O-deoxyhexoside, apigenin$\mathrm{C}$-hexoside-O-pentoside and luteolin-C-hexoside-Opentoside were induced in oats by methyl jasmonate and nematode parasitism. The flavone $\mathrm{C}$-glycoside is toxic to nematodes and supports the view that it is involved in plant defense and in resistance against nematodes.

Previously it has been shown that flavonoids are synthesized by leguminous plants in response to infection with root-knot nematode (Hutangura et al., 1999). But recently it is reported that this process also occurs in non-leguminous plants and in response to cystnematode infection and infection with Xiphinema diversicaudatum, implying that it is likely to be a common response of plants to development of nematode feeding sites of varying ontogeny (Jones et al., 2007). Thus it is believed that flavonoids are inhibitors of local auxin transport and it has been suggested that nematodes induce flavonoid synthesis in order to exploit this property.

\section{Conclusion}

Plants interact with their environment through secondary metabolism. Within this part of the metabolism, the phenylpropanoid pathway is of critical importance as its products (phenolic compounds) protect the plants against abiotic and biotic factors. From the present review it is clearly understood that phenolic compounds have strong potential against nematodes and hence involved in plant defense. So the knowledge of resistance mechanisms characteristic of the resistant plant varieties may facilitate breeding and screening of germplasm and hybrids, or provide a rationale for genetic improvement.

\section{REFERENCES}

Baldridge, G.D., O’Neill, N.R. and Samac, D.A. (1998). Alfalfa (M edicago sativa L.) resistance to the root-lesion nematode, Pratylenchus penetrans: defense response gene mRNA and isoflavonoid phytoalexin levels in roots. Plant Molecular Biology, 38: 999-1010.

Begum, M.Z. and Sivakumar, M. (2004). Assessment of Phenolics and peroxidase activity in Greengram affected by H eter odera cajani and M acrophomina phaseolina. Current Nematology, 15: 73-76.

Begum, S., Wahab, A., Siddiqui, B.S. and Qamar, F. (2000). Nematicidal constituents of the aerial parts of Lantana camara. J ournal of N atural Products, 63: 765-767.

Chen, S., Dickson, D.W. and Hewlett, T.E. (1997). Tannic acid effects on hatching of Heterodera glycines in vitro. Supplementary J ournal of Nematology, 29: 742-745.

Dama, L.B. and Jadhav, B.V. (1997). Antihelminthic effect of juglone on mature and immature $\mathrm{H}$ ymenolepis nana in mice. Rivista di Parassitologia, 2: 303-306.

Dama, L.B. (2002). Effect of naturally occurring napthoquinones on root-knot nematode, Meloidogyne javanica. Indian Phytopathology, 55: 67-69.

Farkas, G.L. and Kiraly, Z. (1962). Role of phenolic compounds in the physiology of plant diseases and disease resistance. 
Phytopathology Zhournal, 44:105-150.

Feeny, P. (1976). Plant apparency and chemical defense. Recent Advances in Phytochemistry, 10: 1-40.

Fetterer, R.H. and Fleming, M.W. (1991). Effect of plumbagin on development of the parasitic nematodes Haemonchus contortus and Ascar is suum. Comparative Biochemistry and Physiology, 100: 539-542.

Ganguly, A.K., Sirohi, A., Pankaj and Singh, V. (1999). Salicylic acid induced resistance in tomato against Meloidogyne incognita race1. Indian J ournal of Nematology, 29: 182184.

Gonzalez, J.A. and Estevez-Braun, A. (1998). Effect of (E)chalcone on potato cyst nematodes (Globodera pallida and G. rostochiensis). Journal of Agricultural and Food Chemistry, 46: 1163-1165.

Hewlett, T.E., Hewlett, E.M. and Dickson, D.W. (1997). Response of Meloidogyne spp., Heterodera glycines and Radopholus similis to tannic acid. Supplementary J ournal of Nematology, 29: 737-741.

Hung, C.L. and Rohde, R.A. (1973). Phenol accumulation related to resistance in tomato to infection by root-knot and lesion nematode. J ournal of Nematology, 5: 255-258.

Hutangura, P., Mathesius, V., Jones, M.G.K. and Rolfe, B.G. (1999). Auxin induction is a trigger for root gall formation caused by root knot nematodes in white clover and is associated with the activation of the flavonoid pathway. Australian J ournal of Plant Physiology, 26: 221-231.

Jayakumar, J., Rajendran, G. and Ramakrishnan, S. (2006). Evaluation of salicylic acid as a systemic resistance inducer against M eloidogyne incognita on tomato cv. Co3. Indian J ournal of Nematology, 36: 77-80.

Jones, J.T., Furlanetto, C. and Phillips, M.S. (2007). The role of flavonoid produced in response to cyst nematode infection of Arabidopsis thaliana. Nematology, 9: 671-677.

Kaplan, D.T., Keen, N.T. and Thomason, I.J. (1980). Studies on the mode of action of glyceollin in soybean incompatibility to the root knot nematode, Meloidogyne incognita. Physiology and Plant Pathology, 16: 319-325.

Kennedy, M.J., Niblack, T.L. and Krishnan, H.B. (1999). Infection by Heterodera glycines elevates isoflavonoid production and influences soybean nodulation. J ournal of Nematology, 51: 341-347.

Laliberte, R., Campbell, D. and Braunderlein, F. (1967). Antihelminthic activities of chalcones and related compounds. Canadian J ournal of Pharmaceutical Sciences, 37-43.

Mahajan, R., Singh, P. and Bajaj, K.L. (1985). Nematicidal activities of some phenolic compounds against M eloidogyne incognita. Revue Nematology, 8, 161-164.

Mahajan, R., Kaur, D.J. and Bajaj, K.L. (1992). Nematicidal activity of phenolic compounds against Meloidogyne incognita. Nematologia M editerranea, 20: 217-219.

Malik, M.S., Pal, V., Sangwan, N.K., Dhindsa, K.S., Verma, K.K. and Bhatti, D.S. (1989). Nematicidal efficacy of substituted phenols, phenoxyacetic acid esters and Hydrazides: A structure-activity relationship study. Nematology, 35: 366-370.

Mian, I.H. and Rodriguez-Kabana, R. (1982). Organic amendments with high tannin and phenolic contents for control of Meloidogyne arenaria in infested soil.
Nematropica, 12: 221-234.

Naik, V.N. and Sharma, D.D. (2007). Efficacy of pesticides and growth hormones against root disease complex of mulberry (M orus al ba L.). International J ournal of Industrial Entomology, 15: 1-6.

Nandi, B., Sukul, N.C. and Babu, S.P.S. (2000a). Exogenous salicylic acid reduces Meloidogyne incognita infestation of tomato. Allelopathy J ournal, 7: 285-288.

Nandi, B., Sukul, N.C., Banerjee, N. and Babu, S.P.S. (2000b). Salicylic acid reduces Meloidogyne infestation of cowpea. Proceedings of Zoological Society, 53: 93-95.

Nandi, B., Sukul, N.C., Banerjee, N., Sengupta, S., Das, P. and Babu, S.P.S. (2002). Salicylic acid enhances resistance in cowpea against Meloidogyne incognita. Phytopathology Mediter ranea, 41: 39-44.

Nandi, B., Kundu, K., Banerjee, N. and Babu, S.P.S. (2003). Salicylic acid induced suppression of M eloidogyne incognita infestation of okra and cowpea. Nematology, 5: 747-752.

Osman, A.A. and Viglierchio, D.R. (1988). Efficacy of biologically active agents as non-traditional nematicides for Meloidogyne javanica. Revue N ematology, 11: 93-98.

Pankaj, Sirohi, A. and Ganguly, A.K. (2001). Partial purification and characterization of 4-hydroxycinnamic acid: COA lyase (EC.6.2.1.12) from resistant and susceptible tomato cultivars inoculated with Meloidogyne incognita. Indian J ournal of Nematology, 31: 105-110.

Pankaj and Sharma, H.K. (2003). Relative sensitivity of Meloidogyne incognita and Rotylenchus reniformis to salicylic acid on okra. Indian J ournal of Nematology, 33: 120-123.

Pankaj, Chawla, G., Shakil, N.A., Kishor, V. and Rohatgi, D. (2005). Estimation of salicylic acid and its role in resistance mechanism in chickpea against $\mathrm{M}$ eloidogyne incognita. Indian J ournal of Nematology, 35: 160-162.

Patil, S.S., Powelson, R.L. and Young, R.R. (1964). Relation of chlorogenic acid and free phenols in potato roots to infection by Verticillium albo-atrum. Phytopathology, 54: 531-535.

Scalbert, A. (1991). Antimicrobial properties of tannins. Phytochemmistry, 30: 3875-3883.

Sirohi, A. and Dasgupta, D.R. (1993). Mechanism of resistance in cowpea to the root-knot nematode, M eloidogyne incognita race 1:1 early induction of phenylalanine ammonia lyase (EC.4.3.1.5) and chlorogenic acid. J ournal of Nematology, 28: $31-41$.

Sirohi, A. and Pankaj. (2005). Bare root dip application of SAR inducing chemicals in tomato against root-knot nematode, Meloidogyne incognita. Indian J ournal of Nematology, 35: 195-198.

Soriano, I.R., Asenstorfer, R.E., Schmidt, O. and Riley, I.T. (2004). Inducible flavone in oats (Avena sativa) is a novel defense against plant-parasitic nematodes. Nematology, 94, 1207-1214.

Stadler, M., Dagne, E. and Anke, H. (1994). Nematicidal activities of two phytoalexins from Taverniera abyssinica. Planta Medica, 60: 550-552.

Swain, S.C., Ganguly, A.K. and Uma R. (2004). Race specific biochemical response in differential hosts against the rootknot nematode, Meloidogyne incognita. Indian J ournal of Nematology, 34: 26-32. 
Thakar, N.A. and Yadav, B.S. (1986). Role of total phenols in pigeonpea resistance to reniform nematode. Indian J ournal of Nematology, 16: 261-262.

Wubben, M.J.E., Jin, J. and Baum, T.J. (2008). Cyst nematode parasitism of A. thaliana is inhibited by salicylic acid (SA) and elicits uncoupled SA-independent pathogenesis-related gene expression in roots. Molecular Plant-Microbe Interactions, 21: 424-432.

Wuyts, N., Waele, D.De. and Swennen, R. (2006a). Activity of phenylalanine ammonia lyase, peroxidase and polyphenol oxidase in roots of banana (Musa acuminata AAA, CVs Grande Naine and Yangambi Km 5) before and after infection with Radopholus similis. Nematology, 8: 201-209.

Wuyts, N., Swennen, R. and Waele, D.De. (2006b). Effects of plant phenylpropanoid pathway products and selected terpenoids and alkaloids on the behavior of the plant-parasitic nematodes Radopholus similis, Pratylenchus penetrans and M eloidogyne incognita. N ematology, 8; 89-101.

Wuyts, N., Lognay, G., Verscheure, M., Marlier, M., Waele, D. De. and Swennen, R. (2007). Potential physical and chemical barrier to infection by the burrowing nematode, Radopholus similis in roots of susceptible and resistant banana (M usa spp.). Plant Physiology, 56: 878-890. 